\title{
Oxidative stress, aging, and diseases
}

This article was published in the following Dove Press journal:

Clinical Interventions in Aging

\author{
Ilaria Liguori' \\ Gennaro Russo' \\ Francesco Curcio' \\ Giulia Bulli' \\ Luisa Aran' \\ David Della-Morte 2,3 \\ Gaetano Gargiulo 4 \\ Gianluca Testa ${ }^{1,5}$ \\ Francesco Cacciatore ${ }^{1,6}$ \\ Domenico Bonaduce' \\ Pasquale Abete' \\ 'Department of Translational \\ Medical Sciences, University of \\ Naples "Federico II", Naples, Italy; \\ 2Department of Systems Medicine, \\ University of Rome Tor Vergata, \\ Rome, Italy; ${ }^{3}$ San Raffaele Roma Open \\ University, Rome, Italy; ${ }^{4}$ Division of \\ Internal Medicine, AOU San Giovanni \\ di Dio e Ruggi di Aragona, Salerno, \\ Italy; ${ }^{5}$ Department of Medicine \\ and Health Sciences, University of \\ Molise, Campobasso, Italy; ${ }^{6}$ Azienda \\ Ospedaliera dei Colli, Monaldi \\ Hospital, Heart Transplantation Unit, \\ Naples, Italy
}

\begin{abstract}
Reactive oxygen and nitrogen species (RONS) are produced by several endogenous and exogenous processes, and their negative effects are neutralized by antioxidant defenses. Oxidative stress occurs from the imbalance between RONS production and these antioxidant defenses. Aging is a process characterized by the progressive loss of tissue and organ function. The oxidative stress theory of aging is based on the hypothesis that age-associated functional losses are due to the accumulation of RONS-induced damages. At the same time, oxidative stress is involved in several age-related conditions (ie, cardiovascular diseases [CVDs], chronic obstructive pulmonary disease, chronic kidney disease, neurodegenerative diseases, and cancer), including sarcopenia and frailty. Different types of oxidative stress biomarkers have been identified and may provide important information about the efficacy of the treatment, guiding the selection of the most effective drugs/dose regimens for patients and, if particularly relevant from a pathophysiological point of view, acting on a specific therapeutic target. Given the important role of oxidative stress in the pathogenesis of many clinical conditions and aging, antioxidant therapy could positively affect the natural history of several diseases, but further investigation is needed to evaluate the real efficacy of these therapeutic interventions. The purpose of this paper is to provide a review of literature on this complex topic of ever increasing interest.
\end{abstract}

Keywords: elderly, reactive oxygen species, reactive nitrogen species, antioxidants

\section{Pathophysiology of oxidative stress}

Free radicals are highly reactive atoms or molecules with one or more unpaired electron(s) in their external shell and can be formed when oxygen interacts with certain molecules. ${ }^{1}$ These radicals can be produced in cells by losing or accepting a single electron, therefore, behaving as oxidants or reductants. ${ }^{2}$ The terms reactive oxygen species (ROS) and reactive nitrogen species (RNS) refer to reactive radical and non-radical derivatives of oxygen and nitrogen, respectively. ${ }^{3}$ Reactive oxygen and nitrogen species (RONS) are produced by all aerobic cells and play an important role in aging as well as in age-related diseases. ${ }^{4}$ RONS generation is not only limited to determine deleterious effects but also involved in the extraction of energy from organic molecules, in immune defense, and in the signaling process..$^{5}$ There are endogenous and exogenous sources of RONS:

- Endogenous sources of RONS include nicotinamide adenine dinucleotide phosphate (NADPH) oxidase, myeloperoxidase (MPO), lipoxygenase, and angiotensin II. ${ }^{6} \mathrm{NADPH}$ oxidase is the prevalent source of the radical superoxide anion $\left(\mathrm{O}_{2}{ }^{\circ}\right)$ which is formed by the one-electron reduction of molecular oxygen, with electrons supplied by NADPH, during cellular respiration. Most of the $\mathrm{O}_{2}$ is dismutated into the hydrogen peroxide $\left(\mathrm{H}_{2} \mathrm{O}_{2}\right)$ by superoxide dismutase (SOD). ${ }^{5}$ $\mathrm{H}_{2} \mathrm{O}_{2}$ is not a free radical because it has no unpaired electrons, but it is able to form the highly reactive ROS hydroxyl ion $\left(\mathrm{OH}^{\bullet}\right)$ through the Fenton or Haber-Weiss
Correspondence: Pasquale Abete Department of Translational Medical Sciences, University of Naples “Federico II", Via S Pansini, Naples 80131 , Italy

Tel +39 08I 7462270

$\mathrm{Fax}+390817462339$

Email p.abete@unina.it 
reaction. Hydroxyl radicals are extremely reactive and react especially with phospholipids in cell membranes and proteins. In neutrophils, $\mathrm{H}_{2} \mathrm{O}_{2}$ in the presence of chloride and MPO can be converted to hypochlorous acid, an ROS particularly damaging cellular proteins. ${ }^{5}$ Nitric oxide (NO) is produced from L-arginine by three main isoforms of nitric oxide synthase (NOS): epithelial NOS, related to vasodilation and vascular regulation, neuronal NOS, linked to intracellular signaling, and inducible NOS, activated in response to various endotoxin or cytokine signals. ${ }^{7}$ Finally, $\mathrm{O}_{2}$ may react with $\mathrm{NO}$ to form another relatively reactive molecule, peroxynitrite $\left(\mathrm{ONOO}^{-}\right){ }^{5,6}$

- Exogenous sources of RONS are air and water pollution, tobacco, alcohol, heavy or transition metals, drugs (eg, cyclosporine, tacrolimus, gentamycin, and bleomycin), industrial solvents, cooking (eg, smoked meat, waste oil, and fat), and radiation, which inside the body are metabolized into free radicals. ${ }^{8}$

RONS, whether they are endogenous or exogenous, cause oxidative modification of each of the major cellular macromolecules (carbohydrates, lipids, proteins, and DNA), ${ }^{6}$ which can also be used as markers of oxidative stress. ${ }^{9}$ Several oxidative changes in proteins have been described. Protein carbonyl (PC) is formed by Fenton reaction of oxidants with lysine, arginine, proline, and threonine residues of the protein side chains. ${ }^{10}$ Carbonyl groups may also derive from the binding of aldehydic lipid oxidation products to lysine, cysteine, or histidine residues called Michael-addition reactions. ${ }^{9}$ Reactions between RNS and tyrosine residues free or within polypeptide sequences induce the formation of nitrotyrosine (NT). ${ }^{9}$ Low-density lipoproteins (LDLs) are the major carriers of cholesterol to body tissues. The oxidation of LDL is a complex process during which both the protein and the lipids undergo oxidative changes that can cause cholesterol accumulation. ${ }^{11}$ Poly-unsaturated fatty acids (PUFAs), in particular linoleic and arachidonic acids, are other important targets of lipid peroxidation, mediated by hydroxyl and peroxyl radicals. Depending on the type of PUFAs undergoing lipid oxidation, several different reactive aldehydes are produced, such as trans-4-hydroxy2-nonenal (4-HNE), malondialdehyde (MDA), and isoprostanes ( $\mathrm{F}_{2}$-IsoPs). ${ }^{9}$ The amino groups of lysine and arginine react with the carbonyl groups of carbohydrates in a process called glycoxidation, resulting in advanced glycation end products (AGEs). Major AGEs include hydroimidazolone, $\mathrm{N} \in$-carboxymethyl-lysine, pentosidine, and glucosepane. ${ }^{12}$ Oxidative damage to DNA results in several mutagenic lesions including 2-hydroxy adenine, 8-oxoadenine, 5-hydroxycytosine, cytosine glycol, thymine, and glycol. The most mutagenic consequences of oxidative stress on DNA are 8-oxo-7,8-dihydro-guanine (8-oxoGuo) and 8-oxo-7,8dihydro- 2 'deoxyguanosine (8-oxodG) lesions, but, among these, the most recurring is 8 -oxoGuo, which can result in G-to-T transversion events. ${ }^{13}$

Antioxidant defense protects biological systems from free radical toxicity and includes both endogenous and exogenous molecules.

- Endogenous antioxidants include enzymatic and nonenzymatic pathways.

The primary antioxidant enzymes are SOD, catalase (CAT), and glutathione peroxidase (GSH-Px). As mentioned above, $\mathrm{O}_{2}$ is converted by SOD to $\mathrm{H}_{2} \mathrm{O}_{2}$, which is decomposed to water and oxygen by CAT, preventing hydroxyl radicals production. Additionally, GSH-Px converts peroxides and hydroxyl radicals into nontoxic forms by the oxidation of reduced glutathione (GSH) into glutathione disulfide and then reduced to $\mathrm{GSH}$ by glutathione reductase. Other antioxidant enzymes are glutathione-S-transferase and glucose-6-phosphate dehydrogenase. ${ }^{14}$

The non-enzymatic antioxidants are molecules that interact with RONS and terminate the free radical chain reactions: bilirubin, $\alpha$-tocopherol (vitamin E), and $\beta$-carotene are present in blood while albumin and uric acid account for $85 \%$ of antioxidant capacity in plasma. ${ }^{15}$

- Exogenous antioxidants include ascorbic acid (vitamin C), which scavenges hydroxyl and superoxide radical anion, $\alpha$-tocopherol (vitamin E), which is involved against lipid peroxidation of cell membranes, and phenolic antioxidants, which include stilbene derivatives (resveratrol, phenolic acids, and flavonoids), oil lecitinas, selenium, zinc, and drugs such as acetylcysteine. ${ }^{16}$ Oxidative stress occurs when there is an imbalance between the formation and the removal of RONS because of an overproduction and/or an impaired ability to neutralize them or to repair the resulting damage. ${ }^{6}$

\section{Oxidative stress and theory of aging}

Aging is the progressive loss of tissue and organ function over time. ${ }^{17}$ The free radical theory of aging, later termed as oxidative stress theory of aging, is based on the structural damage-based hypothesis that age-associated functional losses are due to the accumulation of oxidative damage to macromolecules (lipids, DNA, and proteins) by RONS. ${ }^{18}$ The exact mechanism of oxidative stress-induced aging is 
still not clear, but probably increased RONS levels lead to cellular senescence, a physiological mechanism that stops cellular proliferation in response to damages that occur during replication. Senescent cells acquire an irreversible senescence-associated secretory phenotype (SASP) involving secretion of soluble factors (interleukins, chemokines, and growth factors), degradative enzymes like matrix metalloproteases (MMPs), and insoluble proteins/extracellular matrix (ECM) components. ${ }^{1,19}$ RONS induce cellular senescence acting on various components of SASP:

- regulation of mammalian target of rapamycin complexes' functions; ${ }^{1}$

- production of IL- $1 \alpha$ leading to a proinflammatory state, which increases nuclear factor kappa-B (NFkB) activity and epithelial-mesenchymal transition and tumor metastatic progression; ${ }^{1}$

- induction of MMPs expression, which is associated with age-related and chronic diseases such as cancer, Alzheimer's, atherosclerosis, osteoarthritis, and lung emphysema; ${ }^{1}$

- inhibition of FOXO (Forkhead box) proteins activity, which is involved in insulin/insulin-like growth factor1-mediated protection from oxidative stress; ${ }^{1}$

- reduction of sarco/endoplasmic reticulum $\mathrm{Ca}^{2+}$-ATPase activity leading to cardiac senescence;

- inhibition of sirtuins activity leading to an increased production of RONS by SOD inhibition, a proinflammatory state by preventing their inhibition of tumor necrosis factor alpha (TNF $\alpha)$ and $\mathrm{NF} \kappa \mathrm{B}$, and tumorigenic effect by preventing their inhibitory effect on c-Jun and c-Myc; ${ }^{20}$

- regulation of $\mathrm{p} 16 \mathrm{INK} 4 \mathrm{a} / \mathrm{pRB}$ and $\mathrm{p} 53 / \mathrm{p} 21$ pathways leading to senescence. ${ }^{1}$

\section{Oxidative stress and age-related diseases}

Oxidative stress, cellular senescence, and consequently, SASP factors are involved in several acute and chronic pathological processes, such as CVDs, acute and chronic kidney disease (CKD), neurodegenerative diseases (NDs), macular degeneration (MD), biliary diseases, and cancer. Cardiovascular (CV) risk factors (ie, obesity, diabetes, hypertension, and atherosclerosis) are associated with the inflammatory pathway mediated by IL-1 $\alpha$, IL-6, IL-8, and increased cellular senescence. ${ }^{1}$ Moreover, vascular calcification is linked to an SASP-driven osteoblastic transdifferentiation of senescent smooth muscle cells. In many neurodegenerative conditions, including Alzheimer's disease (AD), brain tissue biopsies show increased levels of p16, MMP, and IL-6. ${ }^{21}$ Chronic obstructive pulmonary disease, biliary cirrhosis, cholangitis, and osteoarthritis share several damaging SASP profiles including IL-6, IL-8, and MMP. ${ }^{1}$ The induction of epithelial to mesenchymal transition mediated by RONS promotes cancer metastasis. ${ }^{22}$ In synthesis, given the close relationship between oxidative stress, inflammation, and aging, the oxidation-inflammatory theory of aging or oxiinflamm-aging has been proposed: aging is a loss of homeostasis due to a chronic oxidative stress that affects especially the regulatory systems, such as nervous, endocrine, and immune systems. The consequent activation of the immune system induces an inflammatory state that creates a vicious circle in which chronic oxidative stress and inflammation feed each other, and consequently, increases the age-related morbidity and mortality. ${ }^{23}$

The connection between oxidative stress and the main age-related diseases is described in the following sections (Figure 1).

\section{Oxidative stress and CVDs}

CVDs are a leading cause of morbidity and mortality in the elderly, and atherosclerosis plays a crucial role as main causal event. ${ }^{24}$ Several studies have proven that heart tolerance to oxidative stress decreases with age because of a reduction in the concentrations of the antioxidant enzymes (ie, GSH-Px and SOD), contributing to the development of $\mathrm{CV}$ alterations. ${ }^{25}$

The evidence currently available links atherosclerosis with oxidized LDL-cholesterol (oxLDL) as the compound mainly responsible for its production, also in elderly. ${ }^{26}$ In fact, different studies showed a significant association between oxLDL and higher arterial stiffness, independent of other traditional CVD risk factors. ${ }^{27}$ A study conducted in 2,944 healthy women (aged 30-79 years) underlined increases in oxLDL levels in plasma after 50 years. ${ }^{28}$ The increase of oxLDL with aging may amplify LDL atherogenicity because of the prooxidant and proinflammatory environments that characterize elderly subjects. ${ }^{26}$ Conversely, data for the InCHIANTI dataset, a 9-year follow-up population-based study, showed no association between higher oxLDLs levels (measured with antibody 4E6) and CVD/cardiac mortality, suggesting that in advanced age, the prognostic information added by oxLDLs might not be significant. ${ }^{29}$ Moreover, the development of oxidative stress contributes to vascular endothelial dysfunction with aging. In healthy adults varying in age, brachial artery flow-mediated dilation is inversely 


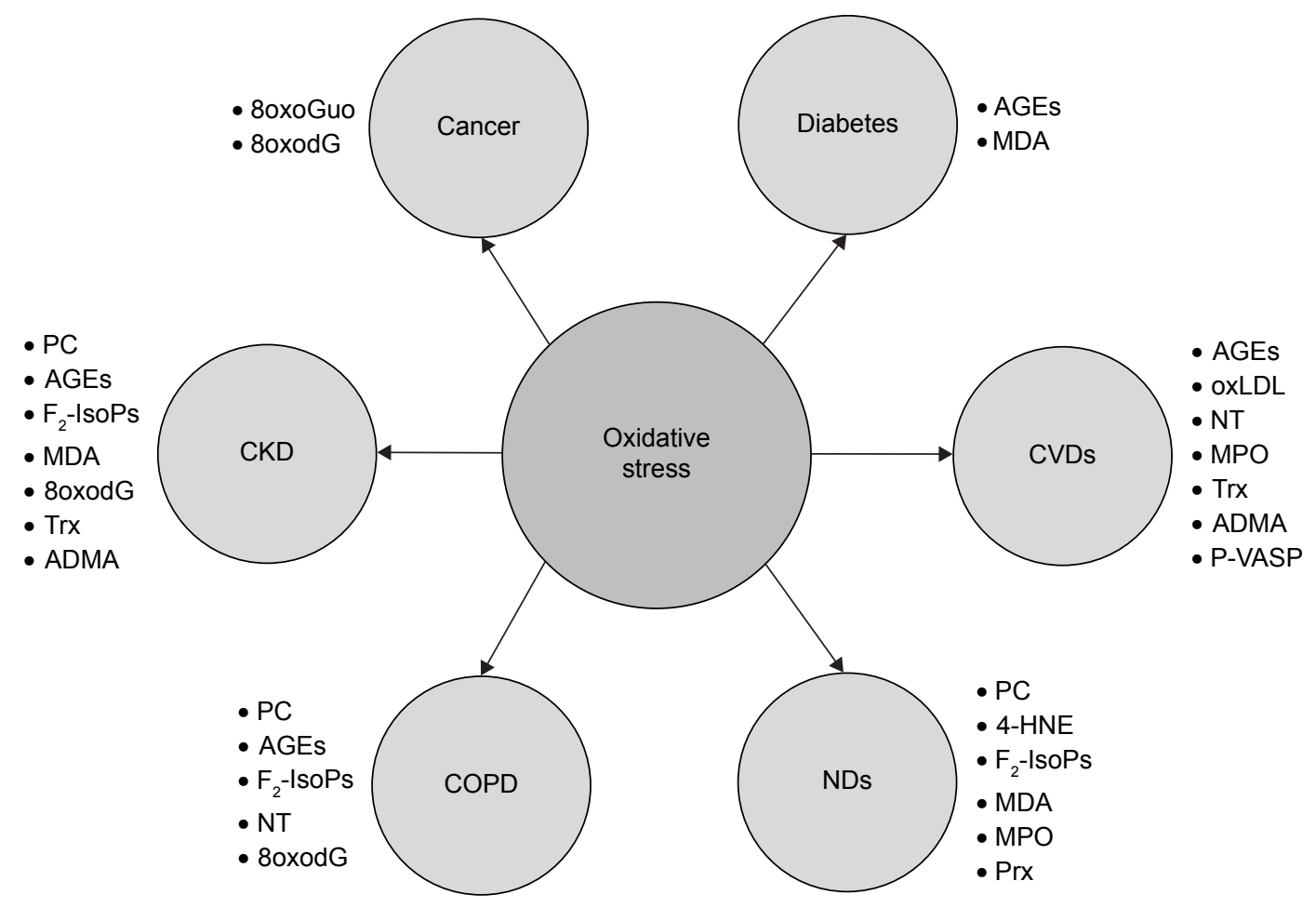

Figure I Oxidative stress, age-related diseases, and relative biomarkers.

Abbreviations: 4-HNE, trans-4-hydroxy-2-nonenal; 8oxodG, 7,8-dihydro-8-oxo-2'-deoxyguanosine; 8oxoGuo, 7,8-dihydro-8-oxoguanosine; ADMA, asymmetric dimethyl L-arginine; AGEs, advanced glycation end products; CKD, chronic kidney disease; CVDs, cardiovascular diseases; $F_{2}$-IsoPs, $F_{2}$-isoprostanes; MDA, malondialdehyde; MPO, myeloperoxidase; NDs, neurodegenerative diseases; NT, nitrotyrosine; oxLDL, oxidized low-density lipoprotein; PC, protein carbonyl; Prx, peroxiredoxins; P-VASP, phosphorylated vasodilator-stimulated phosphoprotein; Trx, thioredoxin.

related to NT in vascular endothelial cells..$^{30}$ Furthermore, the expression of endhotelin-1, the most effective vasoconstrictor molecule produced by the vascular endothelium, is increased in vascular endothelial cells of older compared with younger adults and inversely related to endotheliumdependent dilation and positively related to NT. ${ }^{31}$ Endothelial dysfunction and vascular remodeling are recognized as early determinants in the development of hypertension and atherosclerosis. ${ }^{32}$

\section{Oxidative stress and diabetes}

Diabetes mellitus (type 1 and 2) is a metabolic disease associated with increased formation of free radicals and decreased antioxidant potential, leading to macro- and microvascular complications..$^{33}$ The precise mechanism by which oxidative stress may accelerate the development of complications in diabetes is only partly known. Oxidant stress in type 2 diabetes (T2D) promotes prothrombotic reactions, leading to $\mathrm{CV}$ complications. ${ }^{34}$ Diabetes damages can be considered tissue-oxidative-damaging effects of chronic hyperglycemia. Increased intracellular glucose leads to an increased RONS production, which exceeds the antioxidant capability of the cell to neutralize them. ${ }^{35}$ RONS induce, in this way, the activation of four important molecular pathways involved in hyperglycemia-induced oxidative tissue: activation of protein kinase $\mathrm{C}$ (PKC), increased hexosamine pathway flux, increased AGEs, and increased polyol pathway flux. ${ }^{36}$ In particular, the activation of the AGEs pathway can damage cells modifying the regulation of gene transcription, the signaling between the matrix and other cells and blood proteins (eg, albumin), causing them to bind to AGEs receptors (RAGEs) on macrophages/mesangial cells and increase the production of growth factors and proinflammatory cytokines. ${ }^{37}$ Regarding the geriatric population, a study conducted on 61 elderly subjects has shown increased levels of antioxidants (ie, CAT and GSH-Px) in subjects with impaired glucose tolerance (IGT), compared with healthy controls, and increased levels of oxidative stress biomarkers (ie, MDA) in T2D, but not in IGT group. These results suggest that there is an increased oxidative stress in the elderly T2D patients, which can be partly balanced by increased antioxidant defense system in subjects with IGT. ${ }^{38}$

\section{Oxidative stress and COPD}

COPD is a major cause of morbidity and mortality worldwide ${ }^{39}$ and its prevalence increases with age. ${ }^{40}$ Several 
mechanisms related to aging are potentially involved in its pathogenesis, such as shortened telomere length, cellular and immune senescence, inflammation and also oxidative stress. ${ }^{41}$ RONS in COPD result from both cellular and environmental sources. Environmental oxidants from cigarette smoke activate macrophage and epithelial cells triggering proinflammatory cytokine and chemokine generation, which induce immune response. Released proteases break down connective tissues in the lung, potentially resulting in chronic bronchitis or emphysema. Moreover, excessive RONS released via oxidative bursts of polymorphonuclear leukocytes (PMNs) and alveolar macrophages have been shown to inhibit antiprotease processes and accelerate the degradation of lung tissue. RONS also delay the resolution of inflammation via compromising the phagocytic ability of alveolar macrophages, leading to necrosis and emphysema. Likewise, long-term cigarette smoke exposure impairs PMNs and alveolar macrophage phagocytosis and antigenpresentation functions, which could predispose patients to bacterial/viral infection. ${ }^{42}$ Several studies have shown increased levels of biomarkers of oxidative stress in COPD patients, such as 8oxodG, NT, F2-IsoPs, AGEs, and PC, with a strong correlation with the severity of airflow limitation in COPD elderly patients. ${ }^{41}$ In particular, some authors have shown that several age-related diseases, including COPD, are associated with a skeletal muscle dysfunction. Oxidative stress is a major player in the etiology of this condition in the elderly, and specifically, protein carbonylation was shown to modify the function of key enzymes and structural proteins involved in muscle contractile performance. ${ }^{10}$ Furthermore, a decline in some isoforms of the receptor for AGEs has been detected in COPD elderly patients, and a study has shown that its levels have a significant and independent association with $\mathrm{FEV}_{1}, \mathrm{FEV}_{1} / \mathrm{FVC}$, and diffusing capacity of the lung for carbon monoxide in COPD patients, suggesting that it may be a marker of disease severity and consequently a marker of accelerated aging in this cohort. ${ }^{41,43}$

\section{Oxidative stress and CKD}

Oxidative stress plays a pivotal role in the progression of CKD, through glomerular damage and renal ischemia and, indirectly, with inflammation, hypertension, and endothelial dysfunction. ${ }^{44} \mathrm{CKD}$ patients are in a chronic inflammation state characterized by the activation of PMNs and monocytes. These inflammatory cells increase the secretion of NADPH oxidase and MPO that enhance the formation of ROS.45 Leukocytes of CKD patients produce superoxide anions, which inactivate $\mathrm{NO}$, reducing the ability of blood vessels dilatation that contributes to hypertension. NO levels are also reduced because of the lack of its precursor L-arginine, which is formed from L-citrulline in the kidney. Superoxide anion is also capable of reacting with NO itself forming peroxynitrite, which can oxidize NOS making it unstable. This unstable NOS will further augment superoxide production. ${ }^{46}$ NOS activity is also inhibited by NOS inhibitors, such as the asymmetric dimethyl-arginine (ADMA), which accumulates in $\mathrm{CKD}$ and contributes to hypertension through vasoconstriction. ${ }^{47} \mathrm{CKD}$ patients also have high levels of homocysteine, which raises ROS production, and ADMA, reduces NO synthesis, and increases the risk of CV events. ${ }^{45}$ Endothelial dysfunction, induced by oxidative stress, changes vascular permeability and leads to the entry of LDL cholesterol into intimal layer and increased oxLDL levels, which are involved in the atherosclerosis process, as mentioned earlier. ${ }^{48}$ ADMA-induced endothelial dysfunction leads to proteinuria, a sign of glomerular damage associated, in turn, with proinflammatory cytokines secretion, which increases the production of ROS, worsening renal damage..$^{45}$ Finally, in a state of oxidative stress, the oxidation of red blood cells lipid membranes decreases their elasticity shortening their lifespan and increasing the hemolysis probability. This might explain the cause of anemia in patients with CKD in addition to the reduction of erythropoietin synthesis. ${ }^{49}$ In particular, the global prevalence of CKD is rising, particularly among the elderly population, and $\mathrm{CKD}$ accelerates normal aging and leads to worsening frailty in elderly patients through several mechanisms, in particular, oxidative stress. ${ }^{50}$

\section{Oxidative stress, cognitive impairment, and dementia}

Cognitive impairment and dementia affect life quality and life span in the elderly. Not only NDs, which include AD, Parkinson's disease (PD), Huntington's disease (HD), and amyotrophic lateral sclerosis (ALS), but also vascular dementia have a massive impact especially in aging populations - progressive loss of memory, impairments in the movement, or progressive inability to move. ${ }^{51,52}$ Oxidative stress has been shown to play a pivotal role in the pathophysiology of dementia. ${ }^{53}$ In fact, several studies have evaluated the relationship between the levels of some oxidative stress biomarkers and cognitive function, evaluated with Mini-Mental State Examination (MMSE). A recent study showed that increased oxidative stress biomarkers (ie, MDA, GSH-Px, and PC) correlated with raised levels of inflammatory cytokines and both were associated with low cognitive performance in institutionalized elderly people. ${ }^{54}$ 
Another study showed that, despite age being the principle cause of cognitive decline, cognitive impairment is slower in patients with high GSH-Px activity, but high GSH levels seem to accelerate cognitive decline in the elderly. This seems to be a paradoxical finding because high intracellular GSH should have a protective function against cell damage caused by free radicals. A possible hypothesis is that, as GSH is a substrate of GSH-Px, high GSH levels could be a consequence of the increase in oxidative stress induced by a reduction of GSH-Px activity. ${ }^{55}$ Other theories have been suggested to explain the involvement of oxidative stress in the pathophysiology of NDs. For example, oxidative stress is one of the conditions that induces stress granules (SGs) formation. Nuclear SGs contain heat-shock transcription factor $1 / 2$ and pre-mRNA processing factors while cytoplasmic SGs are composed of proteins and non-translating mRNAs. ${ }^{53}$ Normal cytoplasmic SGs assemble in response to various stressful conditions and are degraded once the stress is absent. SGs can also form super-stable aggregates under pathological conditions - more severe stress, mutations that promote SG assembly or amyloid formation, or mutations that reduce their clearance. ${ }^{56}$ SGs produced in response to acute stress are protective and antiapoptotic. In cognitive impairment, the stress is a chronic condition that cannot be resolved; hence, SGs may interfere with neuronal function by silencing transcripts and by sequestering important proteins such as ribonucleoproteins. ${ }^{53}$ Another hypothesis suggests the role of ROS and redox metals in the pathogenesis of AD. Abnormal homeostasis of bioactive metals could be involved in oxidative stress influencing AD - zinc directly affects amyloid-protein precursor, ${ }^{57}$ and aluminum, zinc, iron, and copper directly bind to amyloid promoting its aggregation. ${ }^{58-60}$ Similarly, the redox metals could promote Tau phosphorylation. ROS, amyloid, and Tau protein affect the activity of N-methyl-D-aspartate (NMDA) receptors, triggering an NMDA-mediated excessive influx of $\mathrm{Ca}^{2+}$ in post-synaptic neurons leading to a cascade of events that increase ROS production, oxidative stress, Tau phosphorylation, and lipid peroxidation, ultimately leading to synaptic dysfunction responsible for AD. ${ }^{61}$

\section{Oxidative stress and cancer}

Many studies have demonstrated the direct relationship between chronic inflammation and carcinogenesis. ${ }^{62}$ The main chemical effectors of these effects of the inflammatory response are free radical species derived from oxidative stress induced by inflammation. RONS may directly or indirectly damage other chemical or structural components in target cells or recruit other inflammatory cells leading to additional RONS production that amplifies the damage. ${ }^{63}$ In particular, RONS and inflammatory cytokines, such as TNF $\alpha$, activate the transcription factor $\mathrm{NF} \kappa \mathrm{B}$, which induces the expression of genes involved in cell proliferation, apoptosis, and carcinogenesis. ${ }^{64}$ Chronic inflammation is also associated with angiogenesis, another characteristic of cancer, because RONS can also increase the expression of transcriptional factors (ie, c-fos and c-jun) involved in neoplastic transformation and enhancement of cancer angiogenesis. ${ }^{63}$ Moreover, macrophages, platelets, fibroblasts, and cancer cells are a major source of angiogenic factors (ie, fibroblast growth factor, vascular endothelial growth factor, and prostaglandins-E1 and E2), which increase the production of RONS and, subsequently, increase the risk of cancer. ${ }^{62}$ The mutagenic/carcinogenic potential of RONS is due to their capability of reacting with DNA and chemically modifying it. In particular, ROSinduced DNA damages can result in transcriptional arrest or induction/replication errors, or genomic instability, which are all associated with carcinogenesis. ${ }^{65}$ Among oxidizedDNA products, 8-oxodG and 8-nitroguanine are considered biomarkers for inflammation-induced carcinogenesis. ${ }^{62}$ There is a dramatic age-dependent escalation in cancer risk, and increased oxidative stress and oxidative stress and its damages over a lifetime may be responsible for this phenomenon. ${ }^{66}$ In fact, there is an accumulation of RONS-induced DNA damages with age, which is confirmed by the progressive and statistically significant increase in the levels of 7,8-dihydro8-oxo- 2 '-deoxyguanosine (8oxodG) observed with aging. ${ }^{62}$ On the basis of these considerations, chronic inflammation and oxidative stress should be considered high risk factors for cancer, especially in elderly people. ${ }^{66}$ Based on these analogies, it should be recommended that elderly people should consume higher antioxidant compounds, but the efficacy of antioxidant therapy in the prevention of carcinogenesis is currently under investigation.

\section{Oxidative stress, sarcopenia, and frailty}

With improved life conditions and the availability of innovative treatments, life expectancy and, consequently, the number of elderly in the population have increased. ${ }^{67}$ Aging is associated with strength deficits that are related to frailty, loss of independence, and physical disability. ${ }^{68}$ Reduction in strength is due to both loss of muscle quantity, referred to as sarcopenia, and loss of muscle strength, termed as dynapenia. ${ }^{69}$ Skeletal muscles consume large quantities of oxygen and can generate a great amount of RONS, whose 
accumulation is thought to be a common determinant in the loss of both muscle quantity and quality, through several mechanisms. ${ }^{70}$ First of all, with advancing age, the antioxidants defenses are compromised, and RONS accumulation induces posttranscriptional modifications, which can be used as biomarkers of oxidative stress, such as the nitration and nitrosylation (ie, NT), carbonylation (ie, PC, 4-HNE), and glycation (ie, AGEs). ${ }^{6}$ Second, RONS contribute to sarcopenia also by increasing proteolysis and decreasing muscle protein synthesis, leading to a reduction in muscle mass quantity. ${ }^{3}$ On the other hand, several mechanisms, acting on the neuromuscular junction, are involved in RONS-mediated reduction of muscle quality and strength. First, RONS reduce acetylcholine release at the synaptic cleft, which could lead to a failure in the generation of an action potential by the sarcolemma. Moreover, persistent oxidative stress may change the morphology of the neuromuscular junction causing a reduction in the innervation and fibers number. At the same time, RONS damage impair excitation-contraction (EC) coupling, leading to a lower release of calcium from the sarcoplasmic reticulum. Finally, RONS induce modification in actin and myosin structures significantly reducing the cross-bridge cycling within the myofibrillar apparatus. ${ }^{69}$ Sarcopenia is an important alteration occurring in the elderly and predicts frailty, poor quality of life, and mortality. ${ }^{70}$ Thus, sarcopenia can be assessed objectively and could be used as a predictor of these adverse health outcomes and a therapeutic target for muscle-building interventions in the elderly. ${ }^{71}$ In particular, sarcopenia is said to be the "biological substrate of physical frailty", 72 and the sarcopenia phenotype should be the central focus of frailty assessment and intervention. ${ }^{71} \mathrm{~A}$ recent systematic review on oxidative stress and frailty showed that preclinical frailty is associated with higher peripheral levels of RONS biomarkers and lower antioxidant parameters. However, due to the cross-sectional design of the study, it was not possible to establish the directionality of the relationships between RONS and frailty, since it could be bidirectional (Figure 2). ${ }^{73}$ Moreover, the Framingham Offspring Study showed increased levels of proinflammatory and oxidative stress biomarkers (ie, $\mathrm{C}$ reactive protein and $\mathrm{F}_{2}$-IsoPs) both in pre-frail and frail elderly subjects. ${ }^{74}$ The same results were described in the elderly German population from the ESTHER Cohort Study. ${ }^{75}$ All these studies defined frailty according to Fried et al, who describe a physical frailty phenotype consisting of the presence of three or more of the following characteristics: unintentional weight loss, weakness, exhaustion, low physical activity, and slowness in motor

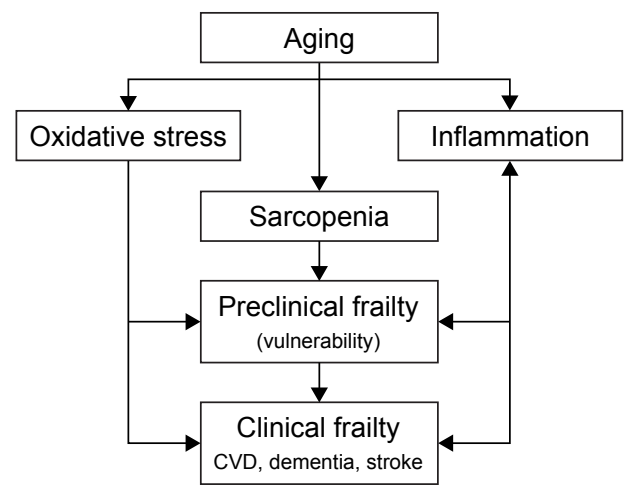

Figure 2 Relationship among oxi-inflamm-aging, preclinical and clinical frailty. Abbreviation: CVD, cardiovascular disease.

performance. ${ }^{76}$ Since Fried's phenotype of frailty focuses on physical frailty, the mechanisms proposed to explain the relationship between oxidative stress and frailty in the elderly are largely overlapped with those described above to explain how oxidative stress causes musculoskeletal system damages. ${ }^{74}$ In other words, frailty in these studies has been defined with criteria of Fried's frailty phenotype, ie, "primary" or "preclinical" frailty, which is not associated with a specific disease. In contrast, "secondary" or "clinical" frailty which is associated with known disability and/or comorbidity (ie, CVDs, NDs, CKD, T2D) ${ }^{77}$ could significantly increase the generation of RONS products. ${ }^{73}$

\section{Biomarkers of oxidative stress}

The World Health Organization defined a biomarker as any substance, structure, or process that can be measured in the body or its products and influence or predict the incidence of outcome or disease. ${ }^{78}$ A clinically useful biomarker should have specificity for a certain disease (diagnostic), have a prognostic value, and correlate with disease activity. ${ }^{9}$ The most clinically relevant oxidative stress biomarkers are described in Table $1^{10,12,26,27,30,31,38,41,43,54,62,66,79-101}$ and Figure 1 and can be divided into RONS-induced modifications, markers of RONS generation, markers of antioxidant defense, and downstream functional markers of RONSinduced damage. ${ }^{9}$

RONS-induced modifications biomarkers include

- PC, whose increased levels are associated with low cognitive performance, ${ }^{54} \mathrm{CKD},{ }^{79} \mathrm{COPD},{ }^{10}$ and sarcopenia $;{ }^{80}$

- AGEs, as mentioned above, derived from glycoxidation reactions. AGEs levels are increased in $\mathrm{T} 2 \mathrm{D}^{12}$ and are an independent risk factor for both all-cause and CV mortality, ${ }^{81}$ also among elderly patients with heart failure. ${ }^{82}$ There is an age-dependent increase of serum 
Table I Overview of the most important oxidative stress biomarkers

\begin{tabular}{|c|c|c|c|}
\hline Biomarkers & Sample & Disease & References \\
\hline \multicolumn{4}{|c|}{ RONS-induced modifications } \\
\hline \multirow[t]{5}{*}{ PC } & Plasma & High levels are associated with raised levels of cytokines and both are associated with & 54 \\
\hline & & low cognitive performance in institutionalized elderly subjects. & \\
\hline & & Higher in CKD. & 79 \\
\hline & & Associated with skeletal muscle dysfunction in elderly COPD patients. & 10 \\
\hline & & Increased levels are associated with sarcopenia in elderly subjects. & 80 \\
\hline \multirow[t]{8}{*}{ AGEs } & Serum & Higher in T2D. & 12 \\
\hline & & $\begin{array}{l}\text { Independent risk factor for both all-cause and cardiovascular disease mortality in } \\
\text { elderly subjects with or without CHF. }\end{array}$ & 81,82 \\
\hline & & Correlate with lipid profiles and atherosclerotic characteristics in the elderly. & 83 \\
\hline & & Higher in elderly subjects with CKD. & 81 \\
\hline & & Higher in elderly subjects with osteoporosis. & 85 \\
\hline & & $\begin{array}{l}\text { Decreased levels of their receptors are markers of severity and accelerated aging in } \\
\text { COPD elderly patients. }\end{array}$ & 43 \\
\hline & & Increased levels are associated with sarcopenia in elderly subjects. & 86 \\
\hline & Skin & $\begin{array}{l}\text { Correlation with risk of exhaustion and low energy expenditure, but not with prevalent } \\
\text { or incident frailty in elderly subjects. }\end{array}$ & 87 \\
\hline \multirow[t]{2}{*}{ oxLDL } & Plasma & High levels are associated with arterial stiffness and atherogenesis in the elderly. & 26 \\
\hline & & Not predictive of cardiac mortality in elderly subject. & 27 \\
\hline \multirow[t]{2}{*}{ 4-HNE } & Plasma & Increased in elderly subjects with $A D, P D, H D$, and ALS pathologies. & 88 \\
\hline & & Increased levels are associated with sarcopenia in elderly subjects. & 89 \\
\hline \multirow[t]{5}{*}{$\mathrm{F}_{2}$-IsoPs } & Urine & High levels are associated with depressed mood in community-dwelling elderly subjects. & 90 \\
\hline & & High levels in AMD. & 91 \\
\hline & & High levels in $A D$ and $H D$. & 92 \\
\hline & & High levels in CKD and correlate with disease progression. & 79 \\
\hline & & High levels in COPD elderly patients. & 41 \\
\hline \multirow[t]{3}{*}{ MDA } & Plasma & $\begin{array}{l}\text { Increased levels correlate with raised levels of inflammatory cytokines and both are } \\
\text { associated with low cognitive performance in institutionalized elderly subjects. }\end{array}$ & 54 \\
\hline & & Increased in elderly subject with T2D but not with IGT. & 38 \\
\hline & & $\begin{array}{l}\text { Elevated in CKD, inversely related to GFR, and positively correlated with uremic toxins } \\
\text { and severity of glomerulosclerosis. }\end{array}$ & 79 \\
\hline \multirow[t]{3}{*}{ NT } & Plasma & Nytrotyrosine levels are associated with vascular endothelial dysfunction with aging. & 30,31 \\
\hline & & High levels in COPD elderly patients. & 41 \\
\hline & Tissue & Increased levels are associated with sarcopenia in elderly subjects. & 93 \\
\hline \multirow[t]{3}{*}{ 8oxodG } & Urine & Markers of inflammation-induced carcinogenesis increasing with aging. & 62 \\
\hline & & High levels in COPD elderly patients. & 41 \\
\hline & & High levels in CKD. & 79 \\
\hline 8oxoGuo & Urine & Markers of carcinogenesis in the elderly. & 66 \\
\hline \multicolumn{4}{|c|}{ Markers of RONS generation } \\
\hline \multirow[t]{3}{*}{ MPO } & Plasma & MPO predicts endothelial dysfunction and the development of $\mathrm{CHF}$ in the elderly. & 94 \\
\hline & & MPO predicts all-cause mortality in frail and very old community-dwelling people. & 95 \\
\hline & & MPO levels are elevated in elderly with $A D$. & 96 \\
\hline \multicolumn{4}{|c|}{ Markers of antioxidant defense } \\
\hline \multirow[t]{2}{*}{ Trx } & Plasma & Significantly increased in CHF compared with healthy elderly subjects. & 97 \\
\hline & & Inverse relationship with GFR, suggesting a protective mechanism. & 79 \\
\hline \multirow[t]{2}{*}{$\operatorname{Prx}$} & Plasma & Potential biomarker of $A D$ in elderly subjects. & 98 \\
\hline & & $\begin{array}{l}\text { Potential useful risk stratification tool to predict mortality in elderly patients with } \\
\text { nonspecific complaints presenting to the emergency department. }\end{array}$ & 99 \\
\hline \multicolumn{4}{|c|}{ Downstream functional markers of RONS-induced damage } \\
\hline \multirow[t]{2}{*}{ ADMA } & Plasma & Strong prognostic value for mortality and future $\mathrm{CV}$ events in the elderly. & 100 \\
\hline & & Elevated in CKD and is an independent predictor of superoxide generation. & 79 \\
\hline P-VASP & Platelets & Establish the efficacy of antiplatelet drugs in the adults and the elderly. & 101 \\
\hline
\end{tabular}

Abbreviations: 4-HNE, trans-4-hydroxy-2-nonenal; 8oxodG, 7,8-dihydro-8-oxo-2'-deoxyguanosine; 8oxoGuo, 7,8-dihydro-8-oxoguanosine; AD, Alzheimer's disease; ADMA, asymmetric dimethyl L-arginine; AGEs, advanced glycation end products; ALS, amyotrophic lateral sclerosis; AMD, age-related macular degeneration; CHF, chronic heart failure; CKD, chronic kidney disease; CV, cardiovascular; $F_{2}$-IsoPs, $F_{2}$-isoprostanes; GFR, glomerular filtration rate; HD, Huntington's disease; IGT, impaired glucose tolerance; MDA, malondialdehyde; MPO, myeloperoxidase; NT, nitrotyrosine; oxLDL, oxidized low-density lipoprotein; PC, protein carbonyl; PD, Parkinson's disease; Prx, peroxiredoxins; P-VASP, phosphorylated vasodilator-stimulated phosphoprotein; RONS, reactive oxygen and nitrogen species; T2D, type 2 diabetes; Trx, thioredoxin. 
AGEs levels, and in the elderly, AGEs levels positively correlate with lipid profiles and atherosclerotic characteristics, suggesting that AGEs could be used as a marker to predict atherosclerotic lesions. ${ }^{83}$ Increased AGEs levels are also associated with chronic kidney disease, predicting a greater decline of renal function. ${ }^{84}$ Accumulation of AGEs in the collagen matrix of bone increases its stiffness and fragility; in fact, AGEs levels are significantly higher in patients with osteoporosis. ${ }^{85}$ Moreover, a recent interesting French study analyzed the relationships between the accumulation of AGEs, assessed by skin autofluorescence (AF), and frailty in 423 older community-dwellers aged $\geq 75$ years, showing that the accumulation of AGEs was not associated with prevalent or incident frailty but with the 4-year risk of exhaustion and low energy expenditure. ${ }^{87}$ Nevertheless, increased levels of AGEs are associated with sarcopenia in elderly subjects, ${ }^{86}$ while decreased levels of RAGEs are markers of severity and accelerated aging in COPD patients; ${ }^{43}$

- oxLDL levels, as described in the section "Oxidative stress and CVDs", correlate with arterial stiffness and atherogenesis in the elderly, not predicting mortality; ${ }^{26,27}$

- 4-HNE levels are elevated in elderly subjects with NDs (ie, AD, PD, HD, and ALS) ${ }^{88}$ and sarcopenia; ${ }^{89}$

- $\mathrm{F}_{2}$-isoprostanes urinary levels are increased in several diseases frequently diagnosed in elderly patients, such as depression, ${ }^{90}$ age-related macular degeneration (AMD), ${ }^{91}$ $\mathrm{AD}$ and $\mathrm{HD},{ }^{92} \mathrm{COPD},{ }^{41}$ and $\mathrm{CKD}$, where it correlates with disease progression; ${ }^{79}$

- MDA increased plasma levels correlate with low cognitive performance in institutionalized elderly subjects $;{ }^{54}$ its levels increase in elderly subjects with T2D but not with $\mathrm{IGT}^{38}$ and in $\mathrm{CKD}$, where its levels are inversely related to glomerular filtration rate (GFR) and positively correlated with uremic toxins and severity of glomerulosclerosis $;{ }^{79}$

- NT levels are used as biomarkers of COPD ${ }^{41}$ and sarcopenia $^{93}$ in elderly subjects, and high levels are associated with vascular endothelial dysfunction with aging ${ }^{30,31}$

- DNA modifications, 8oxodG and 7,8-dihydro-8oxoguanosine (80xoGuo), are excreted into the urine and measured with enzyme-linked immunosorbent assay. Both are markers of inflammation and carcinogenesis in the elderly. ${ }^{62,66}$ Furthermore, 8oxodG is also a marker of $\mathrm{COPD}^{41}$ and CKD in elderly subjects. ${ }^{79}$ In fact, data from the "Osservatorio Geriatrico Campano" showed that high levels of oxidative stress, quantified with 8 oxodH, are associated with high mortality in age-related chronic diseases

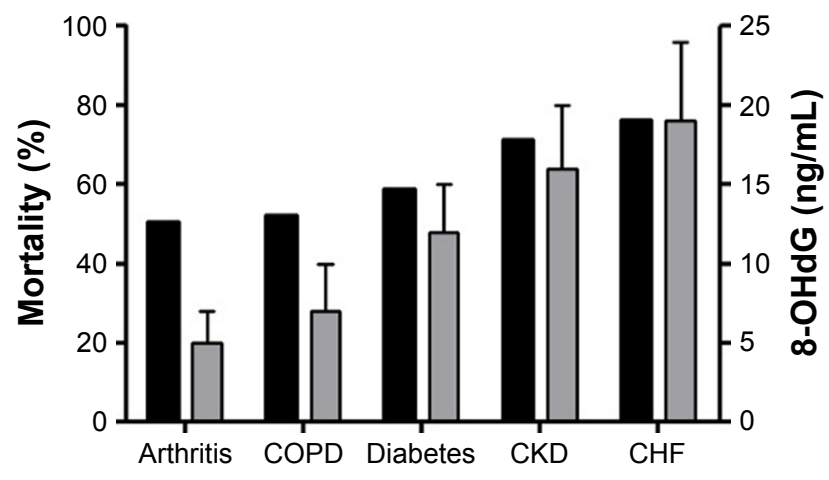

Figure 3 Chronic diseases, oxidative stress, and long-term mortality in communitydwelling elderly subjects. Data from a previous study. ${ }^{102}$

Abbreviations: $\mathrm{CHF}$, chronic heart failure; CKD, chronic kidney disease; 8-OHdG, 8-hydroxy-2'-deoxyguanosine.

(ie, COPD, CKD, chronic heart failure [CHF], and T2D) in community-dwelling elderly subjects (Figure 3 ). ${ }^{102}$

The most important marker of RONS generation is MPO. MPO is a leukocyte-derived enzyme released in inflammatory conditions, which catalyzes the formation of several ROS and is involved in LDL oxidation and is considered a major contributor in the formation and rupture of atherosclerotic plaque. ${ }^{103}$ Moreover, MPO direct catalyzes the consumption of NO resulting in endothelial dysfunction. ${ }^{94}$ Therefore, MPO levels independently predict endothelial dysfunction and mortality for CVDs and CHF. Data from the Aging and Longevity Study in the Sirente Geographic Area (ilSIRENTE Study), a prospective cohort study that collected data on all individuals aged $\geq 80$ years living in a mountain community $(n=363)$, showed that high plasma levels of MPO are positively associated with all-cause death in frail and very old persons living in the community, independent of age, gender, and other clinical and functional variables. In particular, after adjusting for several clinical correlates of mortality risk, baseline MPO plasma concentration emerged as an independent predictor of mortality. ${ }^{95}$ A study conducted on 3,733 elderly subjects measuring MPO levels showed an independent association between increased MPO levels and the development of $\mathrm{CHF}$, particularly beyond myocardial infarction and traditional cardiac risk factors. ${ }^{94}$ Moreover, among age-related diseases, MPO levels are also elevated in elderly subjects with AD, showing that MPO might be an important molecular link between atherosclerosis and $\mathrm{AD}$ and probably a promising biomarker for the detection and risk stratification of $\mathrm{AD}$ patients. ${ }^{96}$

Markers of antioxidant defense include the following:

- Thioredoxin, NADPH, and thioredoxin reductase (TrxR) are important defense systems against oxidative stress, 
providing the electrons to thiol-dependent peroxidases (peroxiredoxins) to remove RONS. ${ }^{104}$ Trx levels are significantly increased in CHF compared with healthy elderly subjects ${ }^{97}$ and its levels show an inverse relationship with GFR, suggesting a protective mechanism. ${ }^{79}$

- Peroxiredoxins are thiol-specific proteins that react as scavenger of $\mathrm{H}_{2} \mathrm{O}_{2}$, peroxynitrite, and a wide range of hydroperoxides. ${ }^{105}$ Peroxiredoxins are potential biomarkers of $\mathrm{AD}$ in elderly subjects ${ }^{98}$ and, interestingly, they could be a useful risk stratification tool to predict mortality in elderly patients with nonspecific complaints presenting to the emergency department. ${ }^{99}$

There are also two markers that reflect oxidative stress downstream of the RONS-induced damage: ADMA and phosphorylated vasodilator-stimulated phosphoprotein (P-VASP). ${ }^{9}$

ADMA is a powerful inhibitor of NOS and also competes with L-arginine for the binding site of this enzyme. Reduced production of endogenous NO coincides with high ADMA levels, engenders endothelial dysfunction, and this dysfunction is reversed by L-arginine. ${ }^{106}$ In particular, in the randomly selected, community-based sample of 1155 elderly, aged 65-102 years, of the "Invecchiare in Chianti Study" (InCHIANTI; aging in the Chianti area), higher ADMA levels independently predicted all-cause and CV mortality. Thus, ADMA could be used as a prognostic value for mortality and future $\mathrm{CV}$ events. ${ }^{100}$

P-VASP is probably the best-established marker for physiological cyclic guanosine monophosphate (cGMP) signaling, because it is phosphorylated mainly by cGMPdependent protein kinases, and lowered P-VASP levels are indicative of pathological signaling. ${ }^{9}$ However, in human blood samples, P-VASP levels are used to establish the efficacy of (or detect nonresponders to) antiplatelet drugs, also in the elderly, as shown in the GENERATIONS trial. ${ }^{101,107}$

Oxidative stress biomarkers may provide important information about the efficacy of a treatment, thus, providing guidance for the selection of the most effective drugs/dose regimens for patients. In addition, if a biomarker of oxidative stress is particularly relevant from a pathophysiological point of view, it may also be useful in research as a therapeutic target to identify novel treatments with antioxidant properties. However, further investigations are needed to confirm and explore their potential clinical applications. ${ }^{9}$

\section{Therapeutic approach}

Since it has been established that oxidative stress plays an important role in the pathogenesis of many clinical conditions and aging, several studies have been conducted to investigate the therapeutic effects of antioxidant therapy.

\section{Vitamins}

The most extensively studied antioxidants are vitamin A, and its precursor $\beta$-carotene, vitamin $C$, and vitamin E. ${ }^{108}$ Several large observational studies were conducted on the effect of intake of different vitamins and on the risk of CVDs, suggesting that higher intake of these vitamins significantly lowered the risk of these pathologic conditions. ${ }^{109}$ Systematic reviews and meta-analysis conducted by Cochrane group investigators to study the effect of vitamins on all-cause mortality showed conflicting and, many times, disappointing results. In some trials, vitamins did not seem to significantly affect mortality, but in several other trials, they were administered alone or in combination showing a significant increase of all-cause mortality. ${ }^{110}$ These conflicting findings can lead to the conclusion that vitamins cannot be used as effective antioxidant therapeutic agents.

\section{Coenzyme Q10}

Coenzyme Q10 (CoQ10), ubiquinone (oxidized form) or ubiquinol (reduced form), is an endogenous lipid that takes part in the mitochondrial respiratory chain reactions. ${ }^{111}$ Numerous pathological processes are associated with primary and secondary CoQ10 deficits, including mitochondrial diseases, fibromyalgia, CVDs, NDs, cancer, T2D, male infertility, and periodontal disease. ${ }^{112} \mathrm{CoQ} 10$ treatment is safe in humans, and new formulations that increase CoQ10 absorption and tissue distribution have been developed. Oral administration of CoQ10 is a frequent antioxidant strategy in many of the abovementioned diseases providing a significant to mild symptomatic benefit. ${ }^{112}$

\section{Selenium}

Many of the physiological roles of the element selenium (Se) are directly attributed to its presence within at least 25 proteins, named selenoproteins, collectively essential for life and also involved in oxidative stress control. ${ }^{113}$ Several selenoproteins have been characterized as antioxidant enzymes, such as GSH-Px, TrxR, and iodothyronine deiodinases. ${ }^{114}$ Several clinical trials have provided convincing evidence of the central role of this element in the prevention and treatment of multiple diseases, such as CVDs, atherosclerosis, diabetes mellitus, stroke, NDs, depression, hypothyroidism, and cancer. ${ }^{115}$ However, there are some controversies over whether Se administration increases the risk of some neuronal diseases (ie, ALS) because of its 
neurotoxic effects - inhibition of prostaglandin D synthase in the brain, inhibition of squalene mono-oxygenase, increase in dopamine and its metabolites, inhibition of succinic dehydrogenase, acetylcholine esterase and $\mathrm{Na}+\mathrm{K}+\mathrm{ATPase}$, and induction of seizures. ${ }^{116}$ Additional experimental evidence is needed to determine the optimal level of Se daily intake that can maximize health benefits, avoiding potential toxic effects. ${ }^{115}$

\section{Polyphenols}

Polyphenols are secondary metabolites of plants, and they are found largely in fruits, vegetables, cereals, and beverages. Typically, a glass of red wine contains about $100 \mathrm{mg}$ polyphenols. ${ }^{117}$ The phenolic compounds of wine can be divided into flavonoids and nonflavonoids. Flavonoids account for $>85 \%$ of the phenolic components in red wine and include different molecules, especially quercetin, and nonflavonoids include mainly resveratrol. ${ }^{118}$ Quercetin is an antioxidant agent present in wine that has been shown to prevent or delay the initiation of CVDs or cancer through different biological effects. Its mechanisms of action are not completely understood, but it has shown an antiinflammatory effect through a decrease of TNF $\alpha$ and IL- $1 \beta$ and suppression of NFKB. ${ }^{119}$ Resveratrol is an antioxidant agent derivative of stilbene, which passed unnoticed until it was related to the French paradox. ${ }^{118}$ The "French paradox" is a term generated in 1992 based on epidemiological data from French people who had a low incidence of CVDs despite the high consumption of saturated fat. This paradox was attributed to their high wine consumption (20-30 g/day), which was suggested to decrease platelet aggregation. ${ }^{120}$ Since 1992, the French paradox has considerably evolved, and its explanation has been attributed to resveratrol beneficial effects because it

- reduces vascular remodeling and inflammation, reducing vascular smooth muscle cells proliferation, vascular calcification, and interfering with the expression of proinflammatory enzymes; ${ }^{118}$

- prevents endothelial dysfunction increasing the expression of NOS and vascular dilation, contributing also to the prevention of hypertension; ${ }^{121}$

- reduces platelet recruitment and aggregation; ${ }^{122}$

- reduces LDL oxidation and LDL and triglycerides levels; ${ }^{118}$

- improves glucose metabolism by increasing insulin sensitivity and glucose absorption mediated by glucose transporter-4 and protecting pancreatic $\beta$-cells function. ${ }^{123}$

These effects are positively related to the reduction of CVDs. However, wine and/or resveratrol can also reduce the incidence of other pathologies, such as neurodegenerative conditions, cancer, and osteoporosis. ${ }^{118}$

\section{Other antioxidant agents}

Many studies have been conducted to identify new natural antioxidants. ${ }^{2}$ In particular, even the fermented papaya preparation (FPP), produced by fermentation of Carica papaya Linn by using yeast, is considered a food supplement that exhibits antiinflammatory, antioxidant, and immunostimulatory functions that could be helpful against age-related and disease-related increase in oxidative stress. In fact, because of the important role of oxidative stress in the pathophysiology of chronic NDs, a study has been conducted to explore the effects of FPP in AD patients, by measuring urinary 8oxodG, showing that, after a supplementation with FPP $4.5 \mathrm{~g} /$ day for 6 months, urinary levels of 8oxodG were significantly decreased $(14.1 \pm 1.7 \mathrm{ng} / \mathrm{mL}$ to $8.45 \pm 1.1 \mathrm{ng} / \mathrm{mL}, p<0.01)$, with no significant changes in controls, showing a potential beneficial effect of FPP in patients with neurodegenerative conditions, such as AD. ${ }^{124}$ Given the reported inverse relationship between the dietary intake of antioxidant-rich food and the incidence of human diseases, there has been a global trend toward increasing the intake of natural antioxidants, especially in the geriatric population., ${ }^{2,125}$ Many antioxidant vegetables and fruits have been identified such as potato, spinach, tomatoes, and legumes or berries, cherries, citrus, prunes, and olives. ${ }^{2,126}$ Interestingly, melatonin is a powerful cardioprotective agent and has shown beneficial effects on cardiac aging and many other age-related disorders. ${ }^{125}$ Melatonin (N-acetyl-5-methoxytryptamine) is an endogenously produced indoleamine synthesized from the essential amino acid tryptophan and secreted by the pineal gland in a circadian manner, but also by extrapineal organs (ie, gastrointestinal tract, immune system cells, retina, spleen, liver, kidney, and heart) not in a circadian manner. ${ }^{127,128}$ Melatonin has also been identified in a very large number of plant species, in plant food and medical herbs. In particular, among the plant products in which melatonin has been identified, the most important are diet products including wine, olive oil, tomato, and beer. ${ }^{125}$ Melatonin acts through several mechanisms: directly scavenging free radicals and indirectly upregulating antioxidant enzymes and downregulating prooxidant enzymes. ${ }^{129}$ Aging is associated with a significant reduction in endogenous melatonin secretion, resulting in an augmentation of oxidative stress and other metabolic changes. ${ }^{130}$ Melatonin receptors have been identified in the human CV system, mainly localized in the ventricular wall, coronary arteries, aorta, and peripheral arteries, where they exert a 
protective effect from serious $\mathrm{CV}$ events, such as ischemic heart disease, acute myocardial infarction, and also cardiac syndrome X, an angina-like chest discomfort. ${ }^{125}$ In particular, some authors have observed an antihypertensive effect of the melatonin, administered orally at $1.5 \mathrm{mg}$ each day, on elderly hypertensive volunteers of both genders (63-91 years old), hypothesizing as a possible explanation that melatonin binds to peripheral receptors in the arteries inducing vasodilatation followed by a decrease in arterial blood pressure. ${ }^{131}$ Melatonin also shows a protective effect from cardiac ischemiareperfusion(RI)-induced infarction. ${ }^{132}$ Furthermore, a recent study showed that the oral administration of melatonin in patients who were undergoing elective coronary artery bypass grafting could ameliorate, in a dose-dependent way (10 mg capsule vs $20 \mathrm{mg}$ capsule once daily), myocardial RI injury by interfering with the oxidative stress, inflammation, and apoptotic markers. ${ }^{133}$ Melatonin may show a protective effect also from myocardial infarction but, at the present time, only two clinical trials, the MARIA and the IMPACT, are currently investigating the effects of melatonin in patients at high risk of acute myocardial infarction. ${ }^{125}$ In the MARIA trial, patients undergoing revascularization for ST-elevation myocardial infarction (STEMI) received intravenous $(51.7 \mu \mathrm{mol})$ and intracoronary $(8.6 \mu \mathrm{mol})$ melatonin immediately before and during percutaneous coronary intervention (PCI) ${ }^{134}$ whereas, in the IMPACT trial, acute myocardial infarction patients will receive an intracoronary ( $1 \mathrm{mg}$ ) and intravenous $(49 \mathrm{mg})$ dose of melatonin. ${ }^{135}$ In particular, the MARIA trial has showed that, when administered via intravenous and intracoronary during primary PCI for STEMI, melatonin had an acceptable safety and tolerability profile, but it did not appear to exert a significant effect on myocardial infarct size measured by magnetic resonance imaging (MRI) and it may have a detrimental effect after STEMI, mainly because it might facilitate left ventricular remodeling. ${ }^{134}$ Given its cardioprotective effects and safety, increased blood melatonin levels could be obtained by moderate and chronic consumption of wine, beer, walnuts, and other food or beverages that contain melatonin, but further investigations are needed to expand its range of applications and also to better understand its specific mechanism(s) to improve CV physiology. ${ }^{125}$

\section{Physical exercise}

Inactivity and aging are known to increase basal RONS concentrations in skeletal muscle, leading to sarcopenia. ${ }^{136} \mathrm{In}$ contrast, regular physical activity is an important determinant in maintaining an optimal state of health, reducing oxidative stress and preventing chronic diseases, but these beneficial effects are related to the features of physical exercise, especially intensity. ${ }^{137}$ The relationship between exercise intensity and oxidative stress adaptation in older adults shows that both inactivity and high-intensity physical exercise are related to increased oxidative stress, whereas moderate intensity exercise is related to a reduction in oxidative stress levels. ${ }^{136}$ In fact, physical exercise training can be divided into acute, endurance, and resistance. In particular, acute exercise induces increased production of free radicals in elderly subjects, together with an increase in antioxidant defenses which, given its age-related decline, seems to be ineffective in neutralizing all of the free radicals produced during exercise, setting up a status of oxidative stress. ${ }^{138-140}$ On the other hand, endurance training induces both a decrease in the production of free radicals and an increase in antioxidant defenses in elderly people. According to the findings in the literature, optimal aerobic training for oxidative/antioxidant balance effects can be achieved with intensities between $50 \%$ and $80 \%$ of $\mathrm{VO}_{2}$ max (the maximum rate of oxygen consumption measured during incremental exercise) and with a frequency of two to three sessions per week. ${ }^{136}$ At the same time, resistance training improves antioxidant defenses in elderly population. ${ }^{141}$ This effect can be achieved by training protocols provided of sufficient volume for each muscle group (3-5 sets, 10 repetitions) and intensities between $50 \%$ and $80 \%$ of one-repetition maximum, the maximum amount of force that can be generated in one maximal contraction. ${ }^{136}$ The role of oxidative stress in age-related sarcopenia suggests the importance of physical activity in limiting this process in elderly subjects and its association with increased RONS generation, but it can also induce an increase in antioxidants defense, positively affecting oxidative/antioxidant balance, if a moderate intensity protocol of endurance or resistance training is chosen, avoiding acute one. ${ }^{142}$

\section{Conclusion}

RONS are produced by several endogenous and exogenous processes. Oxidative stress results from the imbalance between RONS production and antioxidants defense and is primarily involved in "aging theory", in particular in the "oxi-inflamm-aging hypothesis". Oxidative stress is also related to several chronic diseases and, together with chronic inflammation, to sarcopenia and frailty in elderly population. Biomarkers of oxidative stress may be useful as diagnostic tool or therapeutic target. Antioxidant therapy, such as resveratrol and other nutritional compounds, together with moderate aerobic exercise, may positively affect the clinical damage induced by oxidative stress. Further investigations are needed to evaluate the real efficacy of these therapeutic interventions. 


\section{Disclosure}

The authors report no conflicts of interest in this work.

\section{References}

1. Chandrasekaran A, Idelchik MDPS, Melendez JA. Redox control of senescence and age-related disease. Redox Biol. 2017;11:91-102.

2. Lobo V, Patil A, Phatak A, Chandra N. Free radicals, antioxidants and functional foods: impact on human health. Pharmacogn Rev. 2010; 4(8):118-126.

3. Powers SK, Ji LL, Kavazis AN, Jackson MJ. Reactive oxygen species: impact on skeletal muscle. Compr Physiol. 2011;1(2):941-969.

4. Venkataraman K, Khurana S, Tai TC. Oxidative stress in aging-matters of the heart and mind. Int J Mol Sci. 2013;14(9):17897-17925.

5. Genestra M. Oxyl radicals, redox-sensitive signalling cascades and antioxidants. Cell Signal. 2007;19(9):1807-1819.

6. Salisbury D, Bronas U. Reactive oxygen and nitrogen species: impact on endothelial dysfunction. Nurs Res. 2015;64(1):53-66.

7. Adams L, Franco MC, Estevez AG. Reactive nitrogen species in cellular signaling. Exp Biol Med (Maywood). 2015;240(6):711-717.

8. Phaniendra A, Jestadi DB, Periyasamy L. Free radicals: properties, sources, targets, and their implication in various diseases. Indian J Clin Biochem. 2015;30(1):11-26.

9. FrijhoffJ, Winyard PG, Zarkovic N, et al. Clinical relevance of biomarkers of oxidative stress. Antioxid Redox Signal. 2015;23(14):1144-1170.

10. Barreiro E. Role of Protein Carbonylation in Skeletal Muscle Mass Loss Associated with Chronic Conditions. Proteomes. 2016;4(2):18.

11. Trpkovic A, Resanovic I, Stanimirovic J, et al. Oxidized low-density lipoprotein as a biomarker of cardiovascular diseases. Crit Rev Clin Lab Sci. 2015;52(2):70-85.

12. Reynaert NL, Gopal P, Rutten EPA, Wouters EFM, Schalkwijk CG. Advanced glycation end products and their receptor in age-related, non-communicable chronic inflammatory diseases; overview of clinical evidence and potential contributions to disease. Int J Biochem Cell Biol. 2016;81(Pt B):403-418.

13. Jacob KD, Noren Hooten N, Trzeciak AR, Evans MK. Markers of oxidant stress that are clinically relevant in aging and age-related disease. Mech Ageing Dev. 2013;134(3-4):139-157.

14. Birben E, Sahiner UM, Sackesen C, Erzurum S, Kalayci O. Oxidative stress and antioxidant defense. World Allergy Organ J. 2012;5(1): 9-19.

15. Wu JQ, Kosten TR, Zhang XY. Free radicals, antioxidant defense systems, and schizophrenia. Prog Neuropsychopharmacol Biol Psychiatry. 2013;46:200-206.

16. Pisoschi AM, Pop A. The role of antioxidants in the chemistry of oxidative stress: a review. Eur J Med Chem. 2015;97:55-74.

17. Flatt T. A new definition of aging? Front Genet. 2012;3:148.

18. Beckman KB, Ames BN. The free radical theory of aging matures. Physiol Rev. 1998;78(2):547-581.

19. Pole A, Dimri M, Dimri G. Oxidative stress, cellular senescence and ageing. AIMS Mol Sci. 2016;3(3):300-324.

20. Ferrara N, Rinaldi B, Corbi G, et al. Exercise training promotes SIRT1 activity in aged rats. Rejuvenation Res. 2008;11(1):139-150.

21. Burton DGA, Matsubara H, Ikeda K. Pathophysiology of vascular calcification: pivotal role of cellular senescence in vascular smooth muscle cells. Exp Gerontol. 2010;45(11):819-824.

22. Laberge R-M, Awad P, Campisi J, Desprez P-Y. Epithelial-mesenchymal transition induced by senescent fibroblasts. Cancer Microenviron Off J Int Cancer Microenviron Soc. 2012;5(1):39-44.

23. De la Fuente M, Miquel J. An update of the oxidation-inflammation theory of aging: the involvement of the immune system in oxi-inflammaging. Curr Pharm Des. 2009;15(26):3003-3026.

24. Testa G, Cacciatore F, Galizia G, et al. Waist circumference but not body mass index predicts long-term mortality in elderly subjects with chronic heart failure. J Am Geriatr Soc. 2010;58(8):1433-1440.

25. Abete P, Napoli C, Santoro G, et al. Age-related decrease in cardiac tolerance to oxidative stress. J Mol Cell Cardiol. 1999;31(1):227-236.
26. Gradinaru D, Borsa C, Ionescu C, Prada GI. Oxidized LDL and NO synthesis - biomarkers of endothelial dysfunction and ageing. Mech Ageing Dev. 2015;151:101-113.

27. Brinkley TE, Nicklas BJ, Kanaya AM, et al. Plasma oxidized low-density lipoprotein levels and arterial stiffness in older adults: the health, aging, and body composition study. Hypertension. 2009;53(5):846-852.

28. Paik JK, Chae JS, Kang R, Kwon N, Lee S-H, Lee JH. Effect of age on atherogenicity of LDL and inflammatory markers in healthy women. Nutr Metab Cardiovasc Dis. 2013;23(10):967-972.

29. Zuliani G, Morieri ML, Volpato S, et al. Determinants and clinical significance of plasma oxidized LDLs in older individuals. A 9 years follow-up study. Atherosclerosis. 2013;226(1):201-207.

30. Donato AJ, Eskurza I, Silver AE, et al. Direct evidence of endothelial oxidative stress with aging in humans: relation to impaired endotheliumdependent dilation and upregulation of nuclear factor-kappaB. Circ Res. 2007;100(11):1659-1666.

31. Donato AJ, Gano LB, Eskurza I, et al. Vascular endothelial dysfunction with aging: endothelin-1 and endothelial nitric oxide synthase. Am J Physiol Heart Circ Physiol. 2009;297(1):H425-H432.

32. Siti HN, Kamisah Y, Kamsiah J. The role of oxidative stress, antioxidants and vascular inflammation in cardiovascular disease (a review). Vascul Pharmacol. 2015;71:40-56.

33. Bashan N, Kovsan J, Kachko I, Ovadia H, Rudich A. Positive and negative regulation of insulin signaling by reactive oxygen and nitrogen species. Physiol Rev. 2009;89(1):27-71.

34. De Cristofaro R, Rocca B, Vitacolonna E, et al. Lipid and protein oxidation contribute to a prothrombotic state in patients with type 2 diabetes mellitus. J Thromb Haemost. 2003;1(2):250-256.

35. Brownlee M. Biochemistry and molecular cell biology of diabetic complications. Nature. 2001;414(6865):813-820.

36. Golbidi S, Ebadi SA, Laher I. Antioxidants in the treatment of diabetes. Curr Diabetes Rev. 2011;7(2):106-125.

37. Brownlee M. The pathobiology of diabetic complications: a unifying mechanism. Diabetes. 2005;54(6):1615-1625.

38. Atli T, Keven K, Avci A, et al. Oxidative stress and antioxidant status in elderly diabetes mellitus and glucose intolerance patients. Arch Gerontol Geriatr. 2004;39(3):269-275.

39. Murray CJ, Lopez AD. Alternative projections of mortality and disability by cause 1990-2020: global Burden of Disease Study. Lancet Lond Engl. 1997;349(9064):1498-1504.

40. Chapman KR, Mannino DM, Soriano JB, et al. Epidemiology and costs of chronic obstructive pulmonary disease. Eur Respir J. 2006;27(1): 188-207.

41. Choudhury G, MacNee W. Role of inflammation and oxidative stress in the pathology of ageing in COPD: potential therapeutic interventions. COPD. 2017;14(1):122-135.

42. Zuo L, He F, Sergakis GG, et al. Interrelated role of cigarette smoking, oxidative stress, and immune response in COPD and corresponding treatments. Am J Physiol Lung Cell Mol Physiol. 2014;307(3): L205-L218.

43. Gopal P, Reynaert NL, Scheijen JLJM, et al. Association of plasma sRAGE, but not esRAGE with lung function impairment in COPD. Respir Res. 2014;15:24.

44. Balasubramanian S. Progression of chronic kidney disease: mechanisms and interventions in retardation. Apollo Med. 2013;10(1):19-28.

45. Putri AY, Thaha M. Role of oxidative stress on chronic kidney disease progression. Acta Medica Indones. 2014;46(3):244-252.

46. Modlinger PS, Wilcox CS, Aslam S. Nitric oxide, oxidative stress, and progression of chronic renal failure. Semin Nephrol. 2004;24(4): 354-365.

47. Rysz J, Gluba-Brzózka A, Franczyk B, Jabłonowski Z, CiałkowskaRysz A. Novel Biomarkers in the Diagnosis of Chronic Kidney Disease and the Prediction of Its Outcome. Int J Mol Sci. 2017;18(8): pii: E1702.

48. Cachofeiro V, Goicochea M, de Vinuesa SG, Oubiña P, Lahera V, Luño J. Oxidative stress and inflammation, a link between chronic kidney disease and cardiovascular disease. Kidney Int Suppl. 2008;(111): S4-S9. 
49. Thaha M, Yusuf M, Widodo N, et al. Effect of N-acetylcysteine on malondyaldehyde content after iron treatment in chronic kidney disease stage 5D patients. Juntendo Med J. 2012;58(3):238-243.

50. Walker SR, Wagner M, Tangri N. Chronic kidney disease, frailty, and unsuccessful aging: a review. J Ren Nutr. 2014;24(6):364-370.

51. Cacciatore F, Abete P, de Santis D, Longobardi G, Ferrara N, Rengo F. Mortality and blood pressure in elderly people with and without cognitive impairment. Gerontology. 2005;51(1):53-61.

52. Abete P, Della-Morte D, Gargiulo G, et al. Cognitive impairment and cardiovascular diseases in the elderly. A heart-brain continuum hypothesis. Ageing Res Rev. 2014;18:41-52.

53. Chen L, Liu B. Relationships between stress granules, oxidative stress, and neurodegenerative diseases. Oxid Med Cell Longev. 2017;2017: 1809592.

54. Baierle M, Nascimento SN, Moro AM, et al. Relationship between inflammation and oxidative stress and cognitive decline in the institutionalized elderly. Oxid Med Cell Longev. 2015;2015:804198.

55. Revel F, Gilbert T, Roche S, et al. Influence of oxidative stress biomarkers on cognitive decline. J Alzheimers Dis. 2015;45(2): $553-560$.

56. Ramaswami M, Taylor JP, Parker R. Altered ribostasis: RNA-protein granules in degenerative disorders. Cell. 2013;154(4):727-736.

57. Lammich S, Kojro E, Postina R, et al. Constitutive and regulated alpha-secretase cleavage of Alzheimer's amyloid precursor protein by a disintegrin metalloprotease. Proc Natl Acad Sci USA. 1999;96(7): 3922-3927.

58. Bush AI, Pettingell WH, Multhaup G, et al. Rapid induction of Alzheimer a beta amyloid formation by zinc. Science. 1994;265(5177): 1464-1467

59. Tõugu V, Karafin A, Palumaa P. Binding of zinc(II) and copper(II) to the full-length Alzheimer's amyloid-beta peptide. J Neurochem. 2008;104(5):1249-1259.

60. Mantyh PW, Ghilardi JR, Rogers S, et al. Aluminum, iron, and zinc ions promote aggregation of physiological concentrations of beta-amyloid peptide. J Neurochem. 1993;61(3):1171-1174.

61. Tönnies E, Trushina E. Oxidative stress, synaptic dysfunction, and Alzheimer's disease. J Alzheimers Dis. 2017;57(4):1105-1121.

62. Khansari N, Shakiba Y, Mahmoudi M. Chronic inflammation and oxidative stress as a major cause of age-related diseases and cancer. Recent Pat Inflamm Allergy Drug Discov. 2009;3(1):73-80.

63. Federico A, Morgillo F, Tuccillo C, Ciardiello F, Loguercio C. Chronic inflammation and oxidative stress in human carcinogenesis. Int $J$ Cancer. 2007;121(11):2381-2386.

64. Akira S, Kishimoto T. NF-IL6 and NF-kappa B in cytokine gene regulation. Adv Immunol. 1997;65:1-46.

65. Marnett LJ. Oxyradicals and DNA damage. Carcinogenesis. 2000; 21(3):361-370.

66. Olinski R, Siomek A, Rozalski R, et al. Oxidative damage to DNA and antioxidant status in aging and age-related diseases. Acta Biochim Pol. 2007;54(1):11-26.

67. Suzman R, Beard JR, Boerma T, Chatterji S. Health in an ageing world - what do we know? Lancet. 2015;385(9967):484-486.

68. Abete P, Cacciatore F, Ferrara N, et al. Body mass index and preinfarction angina in elderly patients with acute myocardial infarction. $\mathrm{Am} \mathrm{J}$ Clin Nutr. 2003;78(4):796-801.

69. Baumann CW, Kwak D, Liu HM, Thompson LV. Age-induced oxidative stress: how does it influence skeletal muscle quantity and quality? J Appl Physiol Bethesda (1985). 2016;121(5):1047-1052.

70. Gomes MJ, Martinez PF, Pagan LU, et al. Skeletal muscle aging: influence of oxidative stress and physical exercise. Oncotarget. 2017; 8(12):20428-20440.

71. Afilalo J. Conceptual models of frailty: the sarcopenia phenotype. Can J Cardiol. 2016;32(9):1051-1055.

72. Landi F, Calvani R, Cesari M, et al. Sarcopenia as the biological substrate of physical frailty. Clin Geriatr Med. 2015;31(3):367-374.

73. Soysal P, Isik AT, Carvalho AF, et al. Oxidative stress and frailty: a systematic review and synthesis of the best evidence. Maturitas. 2017;99: $66-72$.
74. Liu CK, Lyass A, Larson MG, et al. Biomarkers of oxidative stress are associated with frailty: the Framingham Offspring Study. Age (Dordr). 2016;38(1):1.

75. Saum K-U, Dieffenbach AK, Jansen EHJM, et al. Association between oxidative stress and frailty in an elderly German population: results from the ESTHER cohort study. Gerontology. 2015;61(5):407-415.

76. Fried LP, Tangen CM, Walston J, et al. Frailty in older adults: evidence for a phenotype. J Gerontol A Biol Sci Med Sci. 2001;56(3):M146-M156.

77. Abete P, Basile C, Bulli G, et al. The Italian version of the "frailty index" based on deficits in health: a validation study. Aging Clin Exp Res. 2017;29(5):913-926.

78. Organization WH, Safety IP on C [webpage on the Internet]. Biomarkers in risk assessment: validity and validation. 2001. Available from: http:// www.who.int/iris/handle/10665/42363. Accessed November 29, 2017.

79. Tucker PS, Dalbo VJ, Han T, Kingsley MI. Clinical and research markers of oxidative stress in chronic kidney disease. Biomarkers. 2013; 18(2):103-115

80. Beltran Valls MR, Wilkinson DJ, Narici MV, et al. Protein carbonylation and heat shock proteins in human skeletal muscle: relationships to age and sarcopenia. J Gerontol A Biol Sci Med Sci. 2015;70(2):174-181.

81. Semba RD, Bandinelli S, Sun K, Guralnik JM, Ferrucci L. Plasma carboxymethyl-lysine, an advanced glycation end product, and all-cause and cardiovascular disease mortality in older community-dwelling adults. J Am Geriatr Soc. 2009;57(10):1874-1880.

82. Koyama Y, Takeishi Y, Arimoto T, et al. High serum level of pentosidine, an advanced glycation end product (AGE), is a risk factor of patients with heart failure. J Card Fail. 2007;13(3):199-206.

83. Chang J-B, Chu N-F, Syu J-T, Hsieh A-T, Hung Y-R. Advanced glycation end products (AGEs) in relation to atherosclerotic lipid profiles in middle-aged and elderly diabetic patients. Lipids Health Dis. 2011;10:228.

84. Semba RD, Fink JC, Sun K, Windham BG, Ferrucci L. Serum carboxymethyl-lysine, a dominant advanced glycation end product, is associated with chronic kidney disease: the Baltimore longitudinal study of aging. J Ren Nutr. 2010;20(2):74-81.

85. Sanguineti R, Puddu A, Mach F, Montecucco F, Viviani GL. Advanced glycation end products play adverse proinflammatory activities in osteoporosis. Mediators Inflamm. 2014;2014:975872.

86. Dalal M, Ferrucci L, Sun K, Beck J, Fried LP, Semba RD. Elevated serum advanced glycation end products and poor grip strength in older community-dwelling women. J Gerontol A Biol Sci Med Sci. 2009;64A(1): 132-137.

87. Pilleron S, Rajaobelina K, Tabue Teguo M, et al. Accumulation of advanced glycation end products evaluated by skin autofluorescence and incident frailty in older adults from the Bordeaux Three-City cohort. PLoS One. 2017;12(10): 0186087.

88. Di Domenico F, Tramutola A, Butterfield DA. Role of 4-hydroxy-2nonenal (HNE) in the pathogenesis of alzheimer disease and other selected age-related neurodegenerative disorders. Free Radic Biol Med. 2017;111:253-261

89. Coto Montes A, Boga JA, Bermejo Millo C, et al. Potential early biomarkers of sarcopenia among independent older adults. Maturitas. 2017;104:117-122.

90. Milaneschi Y, Cesari M, Simonsick EM, et al; Health ABC study. Lipid peroxidation and depressed mood in community-dwelling older men and women. PLoS One. 2013;8(6):e65406.

91. Sabanayagam C, Lye WK, Januszewski A, et al. Urinary isoprostane levels and age-related macular degeneration. Invest Ophthalmol Vis Sci. 2017;58(5):2538-2543.

92. Miller E, Morel A, Saso L, Saluk J. Isoprostanes and neuroprostanes as biomarkers of oxidative stress in neurodegenerative diseases. Oxid Med Cell Longev. 2014;2014:572491.

93. Murakami H, Guillet C, Tardif N, et al. Cumulative 3-nitrotyrosine in specific muscle proteins is associated with muscle loss during aging. Exp Gerontol. 2012;47(2):129-135.

94. Tang WHW, Katz R, Brennan M-L, et al. Usefulness of myeloperoxidase levels in healthy elderly subjects to predict risk of developing heart failure. Am J Cardiol. 2009;103(9):1269-1274. 
95. Giovannini S, Onder G, Leeuwenburgh C, et al. Myeloperoxidase levels and mortality in frail community-living elderly individuals. J Gerontol A Biol Sci Med Sci. 2010;65(4):369-376.

96. Tzikas S, Schlak D, Sopova K, et al. Increased myeloperoxidase plasma levels in patients with Alzheimer's disease. J Alzheimers Dis. 2014;39(3):557-564.

97. Jekell A, Hossain A, Alehagen U, Dahlström U, Rosén A. Elevated circulating levels of thioredoxin and stress in chronic heart failure. Eur J Heart Fail. 2004;6(7):883-890.

98. Yoshida Y, Yoshikawa A, Kinumi T, et al. Hydroxyoctadecadienoic acid and oxidatively modified peroxiredoxins in the blood of Alzheimer's disease patients and their potential as biomarkers. Neurobiol Aging. 2009;30(2):174-185.

99. Nickel CH, Messmer AS, Geigy N, et al. Stress markers predict mortality in patients with nonspecific complaints presenting to the emergency department and may be a useful risk stratification tool to support disposition planning. Acad Emerg Med Off J Soc Acad Emerg Med. 2013;20(7):670-679.

100. Pizzarelli F, Maas R, Dattolo P, et al. Asymmetric dimethylarginine predicts survival in the elderly. Age (Dordr). 2013;35(6):2465-2475.

101. Erlinge D, Gurbel PA, James S, et al. Prasugrel $5 \mathrm{mg}$ in the very elderly attenuates platelet inhibition but maintains noninferiority to prasugrel $10 \mathrm{mg}$ in nonelderly patients: the GENERATIONS trial, a pharmacodynamic and pharmacokinetic study in stable coronary artery disease patients. J Am Coll Cardiol. 2013;62(7):577-583.

102. Testa G, Cacciatore F, Galizia G, et al. Charlson comorbidity index does not predict long-term mortality in elderly subjects with chronic heart failure. Age Ageing. 2009;38(6):734-740.

103. Wang J, Tan G-J, Han L-N, Bai Y-Y, He M, Liu H-B. Novel biomarkers for cardiovascular risk prediction. J Geriatr Cardiol. 2017; 14(2):135-150.

104. Lu J, Holmgren A. The thioredoxin antioxidant system. Free Radic Biol Med. 2014;66:75-87.

105. Espinosa-Diez C, Miguel V, Mennerich D, et al. Antioxidant responses and cellular adjustments to oxidative stress. Redox Biol. 2015;6: 183-197.

106. Cardounel AJ, Cui H, Samouilov A, et al. Evidence for the pathophysiological role of endogenous methylarginines in regulation of endothelial NO production and vascular function. J Biol Chem. 2007 282(2):879-887.

107. Geiger J, Teichmann L, Grossmann R, et al. Monitoring of clopidogrel action: comparison of methods. Clin Chem . 2005;51(6):957-965.

108. Cochemé HM, Murphy MP. Can antioxidants be effective therapeutics? Curr Opin Investig Drugs. 2010;11(4):426-431.

109. Firuzi O, Miri R, Tavakkoli M, Saso L. Antioxidant therapy: current status and future prospects. Curr Med Chem. 2011;18(25):3871-3888.

110. Bjelakovic G, Nikolova D, Gluud LL, Simonetti RG, Gluud C. Mortality in randomized trials of antioxidant supplements for primary and secondary prevention: systematic review and meta-analysis. JAMA 2007;297(8):842-857.

111. Dallner G, Sindelar PJ. Regulation of ubiquinone metabolism. Free Radic Biol Med. 2000;29(3-4):285-294.

112. Garrido-Maraver J, Cordero MD, Oropesa-Ávila M, et al. Coenzyme q10 therapy. Mol Syndromol. 2014;5(3-4):187-197.

113. Papp LV, Holmgren A, Khanna KK. Selenium and selenoproteins in health and disease. Antioxid Redox Signal. 2010;12(7):793-795.

114. Reeves MA, Hoffmann PR. The human selenoproteome: recent insights into functions and regulation. Cell Mol Life Sci. 2009;66(15): 2457-2478.

115. Sanmartin C, Plano D, Font M, Palop JA. Selenium and clinical trials: new therapeutic evidence for multiple diseases. Curr Med Chem. 2011; 18(30):4635-4650.

116. Vinceti M, Bonvicini F, Bergomi M, Malagoli C. Possible involvement of overexposure to environmental selenium in the etiology of amyotrophic lateral sclerosis: a short review. Ann Ist Super Sanita. 2010; 46(3):279-283.

117. Pandey KB, Rizvi SI. Plant polyphenols as dietary antioxidants in human health and disease. Oxid Med Cell Longev. 2009;2(5):270-278.
118. Artero A, Artero A, Tarín JJ, Cano A. The impact of moderate wine consumption on health. Maturitas. 2015;80(1):3-13.

119. Chang Y-C, Tsai M-H, Sheu WH-H, Hsieh S-C, Chiang A-N. The therapeutic potential and mechanisms of action of quercetin in relation to lipopolysaccharide-induced sepsis in vitro and in vivo. PLoS One. 2013;8(11):e80744.

120. Renaud S, de Lorgeril M. Wine, alcohol, platelets, and the French paradox for coronary heart disease. Lancet. 1992;339(8808):1523-1526.

121. Toda N, Ayajiki K. Vascular actions of nitric oxide as affected by exposure to alcohol. Alcohol Alcohol. 2010;45(4):347-355.

122. Chen S, Su Y, Wang J. ROS-mediated platelet generation: a microenvironment-dependent manner for megakaryocyte proliferation, differentiation, and maturation. Cell Death Dis. 2013;4:e722.

123. Huang J-P, Huang S-S, Deng J-Y, Chang C-C, Day Y-J, Hung L-M. Insulin and resveratrol act synergistically, preventing cardiac dysfunction in diabetes, but the advantage of resveratrol in diabetics with acute heart attack is antagonized by insulin. Free Radic Biol Med. 2010; 49(11):1710-1721.

124. Barbagallo M, Marotta F, Dominguez LJ. Oxidative stress in patients with Alzheimer's disease: effect of extracts of fermented papaya powder. Mediators Inflamm. 2015;2015:624801.

125. Ferroni P, Della-Morte D, Palmirotta R, et al. Platinum-based compounds and risk for cardiovascular toxicity in the elderly: role of the antioxidants in chemoprevention. Rejuvenation Res. 2011;14(3):293-308.

126. Furuta S, Nishiba Y, Suda I. Fluorometric assay for screening antioxidative activity of vegetables. J Food Sci. 1997;62(3):526-528.

127. Reiter RJ. The pineal gland and melatonin in relation to aging: a summary of the theories and of the data. Exp Gerontol. 1995;30(3-4): $199-212$

128. Acuña-Castroviejo D, Escames G, Venegas C, et al. Extrapineal melatonin: sources, regulation, and potential functions. Cell Mol Life Sci. 2014;71(16):2997-3025.

129. Hardeland R. Melatonin's antioxidant properties: molecular mechanisms. In: Srinivasan V, Brzezinski A, Oter S, Shillcutt SD, editors Melatonin and Melatonergic Drugs in Clinical Practice. New Delhi: Springer India; 2014:17-26.

130. Abete P, Bianco S, Calabrese C, et al. Effects of melatonin in isolated rat papillary muscle. FEBS Lett. 1997;412(1):79-85.

131. Gubin DG, Gubin GD, Gapon LI, Weinert D. Daily melatonin administration attenuates age-dependent disturbances of cardiovascular rhythms. Curr Aging Sci. 2016;9(1):5-13.

132. Sahna E, Acet A, Kaya Ozer M, Olmez E. Myocardial ischemiareperfusion in rats: reduction of infarct size by either supplemental physiological or pharmacological doses of melatonin. J Pineal Res. 2002;33(4):234-238.

133. Dwaich KH, Al-Amran FGY, Al-Sheibani BIM, Al-Aubaidy HA Melatonin effects on myocardial ischemia-reperfusion injury: impact on the outcome in patients undergoing coronary artery bypass grafting surgery. Int J Cardiol. 2016;221:977-986.

134. Dominguez-Rodriguez A, Abreu-GonzalezP, de la Torre-Hernandez JM, et al; MARIA Investigators. Effect of intravenous and intracoronary melatonin as an adjunct to primary percutaneous coronary intervention for acute ST-elevation myocardial infarction: Results of the Melatonin Adjunct in the acute myocaRdial Infarction treated with Angioplasty trial. J Pineal Res. 2017;62(1): Epub 2016 Nov 5.

135. Halladin N, Ekeløf Busch S, Jensen SE, et al. Intracoronary and systemic melatonin to patients with acute myocardial infarction: protocol for the IMPACT trial. 2014;61(2):A4773.

136. Bouzid MA, Filaire E, McCall A, Fabre C. Radical oxygen species, exercise and aging: an update. Sports Med. 2015;45(9):1245-1261.

137. Radak Z, Zhao Z, Koltai E, Ohno H, Atalay M. Oxygen consumption and usage during physical exercise: the balance between oxidative stress and ROS-dependent adaptive signaling. Antioxid Redox Signal. 2013;18(10):1208-1246.

138. Ji L, Wei Y, Jiang T, Wang S. Correlation of Nrf2, NQO1, MRP1, cmyc and p53 in colorectal cancer and their relationships to clinicopathologic features and survival. Int J Clin Exp Pathol. 2014;7(3): 1124-1131. 
139. Guemouri L, Artur Y, Herbeth B, Jeandel C, Cuny G, Siest G. Biological variability of superoxide dismutase, glutathione peroxidase, and catalase in blood. Clin Chem. 1991;37(11):1932-1937.

140. Fatouros IG, Jamurtas AZ, Villiotou V, et al. Oxidative stress responses in older men during endurance training and detraining. Med Sci Sports Exerc. 2004;36(12):2065-2072.
141. McBride JM, Kraemer WJ, Triplett-McBride T, Sebastianelli W. Effect of resistance exercise on free radical production. Med Sci Sports Exerc. 1998;30(1):67-72.

142. Brioche T, Lemoine-Morel S. Oxidative stress, sarcopenia, antioxidant strategies and exercise: molecular aspects. Curr Pharm Des. 2016; 22(18):2664-2678.

\section{Publish your work in this journal}

Clinical Interventions in Aging is an international, peer-reviewed journal focusing on evidence-based reports on the value or lack thereof of treatments intended to prevent or delay the onset of maladaptive correlates of aging in human beings. This journal is indexed on PubMed Central, MedLine,

\section{Dovepress}

CAS, Scopus and the Elsevier Bibliographic databases. The manuscript management system is completely online and includes a very quick and fair peer-review system, which is all easy to use. Visit http://www.dovepress. com/testimonials.php to read real quotes from published authors. 